\title{
Activities as a Gateway to Sustained Subjective Well-Being Mediated by Products
}

\author{
Lisa Wiese \\ eBay, brands4friends \\ Berlin, Germany \\ l.wiese@tudelft.nl
}

\author{
Anna Pohlmeyer \\ Delft University of Technology \\ Delft, The Netherlands \\ A.E.Pohlmeyer@tudelft.nl
}

\author{
Paul Hekkert \\ Delft University of Technology \\ Delft, The Netherlands \\ P.P.M.Hekkert@tudelft.nl
}

\begin{abstract}
Research in positive psychology indicates that sustained well-being is more determined by our actions than by our possessions. Products' contribution to well-being may thus be grounded in their potential to support well-being-enhancing activities rather than in their material value. In a laddering study, we investigated how products shape a range of well-being determinants, including activities, and well-being outcomes. Following a hierarchical structure, seven product experience qualities, six motivations, and seven activities were empirically found to be linked to long-term well-being. We describe these ingredients for sustained well-being in further detail and provide actionable guidance on how to address them by means of design. As the majority of productsupported long-term well-being outcomes were mediated by activities, we propose activities as most promising starting point in design for sustained well-being.
\end{abstract}

\section{Author Keywords}

$\mathrm{HCI}$; design; user experience; well-being; positive design; experience design

\section{CSS Concepts}

- HCI theory, concepts, models

\section{INTRODUCTION}

Due to technological advancements in recent decades, more and more aspects of our everyday lives are nowadays supported or accompanied by products and services, which creates the opportunity for designers to impact peoples' quality of life and well-being on a broad scale. In fact, some of the most widely used interactive products like social networks and communication technologies explicitly aim to improve core elements of psychological well-being such as having strong and intimate relationships with others [57]. Despite this great potential for positive impact, there is evidence linking specific ways of using interactive technology to impoverished social lives, e.g. reduced quality of face-to-face interactions, decreased measures of mental health, e.g. higher

This work is licensed under a Creative Commons Attribution International 4.0 License.

DIS '19, June 23-28, 2019, San Diego, CA, USA (C) 2019 Copyright is held by the owner/author(s). ACM ISBN 978-1-4503-5850-7/19/06.

https://doi.org/10.1145/3322276.3322297 stress levels, and lower psychological functioning, e.g. increased loneliness (see [8] for an overview). This shows that the effects of technology use can reach far beyond mere product interactions, and create lasting effects on individuals and society. Consequently, design efforts should not stop at shaping human-product interactions in the present but also consider long-term consequences on well-being. Growing awareness of potential risks has lead influential IT corporations to address Digital Wellbeing (Google, e.g. [38]) and Digital Wellness (Apple, e.g. [20]) in their product development efforts.

In parallel to these developments in industry, academic research on well-being design gained momentum within $\mathrm{Hu}-$ man-Computer-Interaction (HCI) in recent years. Publications in this field contributed theoretically informed frameworks [[6],[13],[27],[42],[51]] grounded in psychology, and outlined possibilities to deliberately design for sustained well-being [[44],[47]]. These approaches cover a wider range of psychological or eudaimonic well-being outcomes and emphasize the potential of products to mediate well-being-enhancing activities in the longer term. Some authors also explicitly point to potential negative side-effects that need to be taken into account when designing for well-being [13]. In their line of thinking, design that fosters engagement or triggers positive emotions in a given moment, but, for instance, makes individuals addicted in the long run cannot be considered design for well-being.

However, in order to shape the design of everyday products and technologies, these academic contributions have yet to be translated into actionable design practices and measurement tools that can be applied in industry contexts [[28],[42]]. What is missing is a clear understanding of (causal) relations between product experience qualities, well-being determinants and well-being outcomes in order to address these relations in the design process. In this paper, we will contribute to this understanding by enriching prevailing theoretical models in the field of well-being design with empirical insights. We do so by means of laddering interviews, which illustrate how specific product experiences are linked to (sustained) well-being. Furthermore, we visualize pathways from product experience qualities to well-being determinants and well-being outcomes. To show how product experience qualities are supported by concrete product attributes, we also refer to various ways in which products seem to support these experience qualities. 


\section{RELATED WORK}

Momentary, hedonic aspects of well-being such as pleasure and positive emotions have been studied intensely in the context of human-product interactions (see [14] for an overview). Other more enduring aspects of eudaimonic or psychological well-being (i.e., optimal psychological functioning) $[[53],[56]-[58]]$ such as having a sense of purpose in life, being fully engaged in one's daily activities, and growing as a person, have not explicitly been addressed by design research until the beginning of this decade [[12],[24],[45]] (see [30] for a more systematic definition of hedonia and eudaimonia). Related theoretical frameworks comprise work on Positive Technologies [51], Experience Design [27], Positive Design [13] and Positive Computing [6]. They draw from existing theories in (positive) psychology and specify determinants of psychological well-being that can be tackled by design, e.g. fulfillment of psychological needs [[27],[42]], realization of self-concordant goals [13], personal values [40] and assignment of rewards [6]. Empirical studies on eudaimonic product experiences [[35],[37],[40]] support the involvement of these theoretically suggested determinants in human-product-relationships. However, empirical research in this field is scarce and the exact nature of those relationships is poorly understood, e.g. what are distinct qualities of eudaimonic and hedonic product experiences. Even though these conceptualizations provide a broad theoretical basis for designers to draw from, they remain rather unspecific. In the current study, we will therefore take an exploratory, bottomup-top-down approach to investigate well-being determinants and well-being outcomes empirically found to be supported in human-product-relationships and map these empirical insights with established theories and frameworks in psychology and HCI (gap 1). As requested by Peters et al. [42], our objective is to take first steps towards establishing a library of empirically validated determinants shaping longterm well-being outcomes from human-product-relationships (goal 1).

Well-being design frameworks emphasize nuanced ways in which products contribute to individuals' well-being: (1) directly during human-product interactions and (2) indirectly by mediating, i.e. supporting or enabling positive and meaningful activities [[16],[42],[45]]. On the indirect pathway, the product itself may no longer be in the focus of attention [16] while performing the activity. Referring to research in positive psychology [[34],[62]], activities have been proposed as a particularly promising starting point when designing for sustained well-being [[44],[47]]. This line of research demonstrated that a significant proportion of inter-individual differences in well-being cannot be explained by genetics or life circumstances but by deliberate engagement in well-being-enhancing activities [[34],[62]]. Consequently, numerous cognitive (e.g. expressing gratitude, savoring life's joys), volitional (e.g. committing to one's goals) and behavioral (e.g. learning a new skill, practicing random acts of kindness) activities were empirically studied and found to be linked to lasting increases in well-being [[5],[32],[49],[60]]. Literature points to a number of reasons why activities have these favorable effects on individuals' well-being. One reason is connected to the phenomenon of hedonic adaptation [18] describing how people react to positive changes in their life, e.g. winning the lottery [4], getting married [31] or starting a new job [2] by reverting to their individual happiness baseline level. This mechanism can be compared to running on a 'hedonic treadmill' [3] and was found to arise particularly fast following material acquisitions compared to changes of one's experiential patterns. In addition, material purchases were found to lead to smaller increases in well-being compared to experiential purchases (see [41] for an overview), e.g. because they are more likely to trigger social comparisons. Experiences on the other hand are phenomenologically unique to a person and may serve as means to identity construction and personal storytelling, e.g. describing oneself to other people by referring to personally relevant experiences. Compared to changes in material possessions or one's life circumstances, activities are per se more transient and varied and thus less prone to adaptation [34]. Moreover, engaging in specific activities such as practicing gratitude or savoring life's joys can counterbalance hedonic adaptation [62]. Another promising way to achieve enduring changes in wellbeing, is to establish habits around well-being-boosting activities [34]. Research has further identified specific person characteristics (e.g. motivation, self-efficacy beliefs) and activity characteristics (e.g. dosage, variety) moderating the success of positive activities in terms of well-being enhancements [[33],[34]]. Even though theory and research suggests the importance of activities for sustainable well-being, few empirical studies have been devoted so far to the question if and how products support well-being-increasing activities beyond (the obvious) affordances, e.g. a ball is for kicking and throwing (gap 2). One explicit aim of the current work is therefore to derive a clearer understanding of the contribution of product-mediated activities to long-term well-being (goal 2).

Previous empirical studies [[26],[35],[37],[39],[65]] used online accounts of qualitative, personal narratives to investigate positive and meaningful experiences mediated by products. However, these studies rarely analyzed the content of the qualitative narratives themselves in greater detail or reported difficulties in doing so due to the heterogeneous nature of user-generated narratives gathered in online studies [26]. Consequently, major empirical insights derived from this line of research were primarily based on correlation analysis using quantitative ratings of product-mediated experiences rather than on a qualitative analysis of the personal narratives [65]. Hence, several HCI researchers call for more systematic qualitative analysis techniques to further investigate the essence of positive and meaningful product experiences [[26],[37],[39],[65]].

\section{METHODS \& MATERIALS}

To investigate (causal) relations between product experience qualities, well-being determinants and well-being outcomes 
more systematically, we conducted one-on-one, in-depth laddering interviews following the guidelines by Reynolds \& Gutman [50]. Participants were probed about personal items and a meditation app. Interview data were analyzed based on means-end analysis [[22],[50]]. In order to help participants reflect on their well-being and products' potential contribution, a sensitizing booklet containing daily assignments was sent to participants prior to the interviews.

\section{Participants}

Due to the complexity of laddering interviews and the associated qualitative data analysis, we started with a small participant sample. 14 participants were recruited from the internal database of a German market research company. Two participants were excluded because they did not return the sensitizing booklet in time or did not show up at the interview. The data of the remaining 12 participants (median age: 31 years; range: 18-36 years; 8 female; higher education) was analyzed. Participants were video-recorded during the interviews. Written informed consent was obtained prior to the study and participants were compensated for their efforts (230 Euros).

\section{Materials}

The relationship between product experience qualities, wellbeing determinants and well-being outcomes was examined using two types of products: a) personal items that participants selected based on a sensitizing booklet, and b) a wellbeing product used by all participants (meditation app). Personal items were expected to inform about product categories subjectively associated with well-being and to facilitate participants' reflection about linkages between product experience qualities and well-being outcomes. The meditation app was chosen to assure that the sample included at least one product used by every participant to support pattern extraction from the laddering interviews.

\section{Personal Items (self-selected)}

Participants were asked to bring three products to the interview that they believed contribute to their well-being. They were further instructed to think of products as any kind of object, tool, service, or interactive experience that is created or designed by a human being (not a rock or other natural object, but websites and apps count). This instruction was used in a conference workshop [48] before and found to generate a diverse range of products. In order to assist participants in reflecting on their well-being and select appropriate products, a sensitizing booklet was sent to participants via mail one week prior to the interviews (see [59]), containing 5-10 minutes long assignments (e.g., "Describe your recipe for a happy life", "What was the nicest gift you ever received for your birthday?", "What products would you bring along if you were going to spend a long period of time on a faraway island?") over the course of seven days (presented in German). At the end of the sensitizing period, participants were instructed to select three personal products that they believe contribute to their well-being and bring these items to the interviews.

\section{Well-Being Product (assigned)}

In order to extract patterns across participants based on just a small sample, we further integrated one product all participants were familiar with. The meditation app Headspace (headspace.com) was chosen since it has been linked to improved well-being measures in smartphone-based mindfulness intervention studies [[1],[15]]. All participants were regular users of the app (i.e., have used the app for at least six months; mean: 12.15 ; SD: 7.45 months). Ten participants reported to also use other mental health or well-being apps (e.g. yoga, running, nutrition) regularly.

\section{Procedure}

In order to link product experience qualities to well-being determinants and well-being outcomes, laddering interviews were conducted. Laddering is based on the assumption that consumers choose a product because they suspect specific attributes ('means') lead to beneficial consequences with regard to their personal values ('ends') [22]. Laddering combines specific interviewing techniques with a data analysis format to extract means-end chains (MEC) or ladders from the interview data [50]. MECs represent hierarchical sequences of product attributes (A), perceived consequences (C) and underlying personal values and goals (V), which increase in their abstractness from tangible product features to more general intrapersonal concepts such as motivational consequences and overarching personal values (Figure 1). The laddering interviews (90 minutes) were conducted at a user research facility in Berlin (Germany) and based on a semi-structured interview guide. To minimize order and familiarization effects, participants were first interviewed either about (a) their personal items or (b) the meditation app (randomly assigned). In order to extract MECs, the interviews started with eliciting key product attributes, i.e. concrete and/or experienced product qualities by asking participants (a) what is special about the product, (b) what they like about the product, and (c) which features they would not want to miss about the product. Since products' contribution to well-being was not expected to be easily accessible by participants, we encouraged them to further think about typical positive experiences associated with the product. This usually resulted in nominating motivational and behavioral consequences of product usage and facilitated generating insights about product-mediated activities. The link to personal values was derived by asking participants what these positive experiences meant to them.

\section{Data Analysis}

Video recordings from the interviews were transcribed and analyzed following a four steps approach: (a) content analysis to generate an empirically derived category system, (b) extraction of MECs by linking attributes, consequences and values from individually reported experiences, (c) aggregation of individual MECs into an Implication Matrix (IM) to extract prominent MECs across participants, and (d) visualization of the most prominent MECs as Hierarchical Value Map (HVM). 


\section{Content Analysis}

Interviews were transcribed and analyzed using qualitative open and axial coding [9]. During content analysis, a category system was generated based on participant responses that reflect recurrent key elements related to attributes (A), consequences (C) and values (V) and iteratively refined. Overall, 40 key elements were identified (Table 1). When possible, categorization and terminology were derived from existing theoretical frameworks in (positive) psychology and well-being design to incorporate prevalent knowledge in these respective fields (see Discussion). The aim was to establish a category structure that is neither too narrow (i.e., does not allow abstraction from individual responses) nor too broad (i.e., discards meaningful categories). The resulting category system is based on empirical input from the interviews (bottom-up) and theoretical considerations stemming from established frameworks (top-down); see Table 1 and Discussion.

$\begin{array}{lll}\text { Experience Qualities } & \text { (14) External Pushes } & \text { (27) Relaxation } \\ \text { (1) Context-Sensitivity } & \text { (15) Rewards } & \text { (28) Security, Health } \\ \text { (2) Ease of Use } & \text { Activities } & \text { (29) Self-Actualization } \\ \text { (3) Identification } & \text { (16) Contributing to the Greater Good } & \text { (30) Self-Direction } \\ \text { (4) Joy of Use } & \text { (17) Managing Stress, Hardship, Trauma } & \text { (31) Self-Esteem } \\ \text { (5) Moral Value } & \text { (18) Committing to One's Goals } & \text { (32) Stimulation } \\ \text { (6) Optimal Challenge } & \text { (19) Taking Care of Body \& Mind } & \text { Well-Being Outcomes } \\ \text { (7) Personal Relevance } & \text { (20) Investing in Social Connection } & \text { (33) Comfort } \\ \text { (8) Protection } & \text { (21) Learning } & \text { (34) Engagement } \\ \text { (9) Symbolic Value } & \text { (22) Living in the Present } & \text { (35) Mastery } \\ \text { Motivations } & \text { Intrapersonal Orientations } & \text { (36) Meaning } \\ \text { (10) Autonomy } & \text { (23) Affiliation } & \text { (37) Personal Growth } \\ \text { (11) Broaden Attention } & \text { (24) Benevolence/Universalism } & \text { (38) Positive Emotions } \\ \text { (12) Competence } & \text { (25) Hedonism } & \text { (39) Positive Relationships } \\ \text { (13) Concentration } & \text { (26) Power } & \text { (40) Virtue }\end{array}$

Table 1. Key elements resulting from content analysis

The generated categories were additionally clustered into different hierarchical levels following the A-C-V sequence proposed by Means-End Theory [[22],[50]]. We further derived more than the three basic levels (i.e., attributes, consequences, and values) as advised for the application of MeansEnd Theory in HCI contexts [66] (see Figure 1). More specifically, we differentiated the Attributes (A) level into concrete attributes (CA) and more abstract, experienced product qualities (EQ) to be able to extract patterns across product categories (via EQ) while keeping tangible information (via CA) to help designers deduce actionable insights for product design. Experience qualities indicate how a product (and its attributes) is perceived by an individual [23]. The Consequences level was distinguished into immediate motivational consequences (MO) of product usage and product-mediated activities (AV) promoted by these motivations. We added product-mediated activities as separate level because specific types of activities were hypothesized to play a crucial role in mediating durable well-being outcomes supported by products (goal 2, see above). Referring to the well-being literature (see [29] for a conceptual overview), the Values level was further differentiated into intrapersonal orientations (IO) and well-being outcomes (WB). Intrapersonal orientations re- flect psychological variables within the individual that ultimately drive their behavior (i.e., universal needs, personal goals, personal values). Well-being outcomes comprise (subjective) experiences and aspects of psychological functioning, i.e. how well a person is doing in life. For each product experience we further captured whether reported increases in well-being were momentary (short-term) or persisted over longer periods of time (long-term).

\section{Means-End Chains}

Based on the generated category system (Table 1) and the category structure outlined above (see also Figure 1), MECs were composed for each participant individually by scanning transcripts for coherent experiential episodes containing one or multiple MECs: 115 individual product experiences were analyzed overall; 51 of them were linked to personal items and 64 of them to the meditation app. The basic structure of MECs (left, middle), as well as an example MEC from an individual product experience can be found in Figure 1 (right).

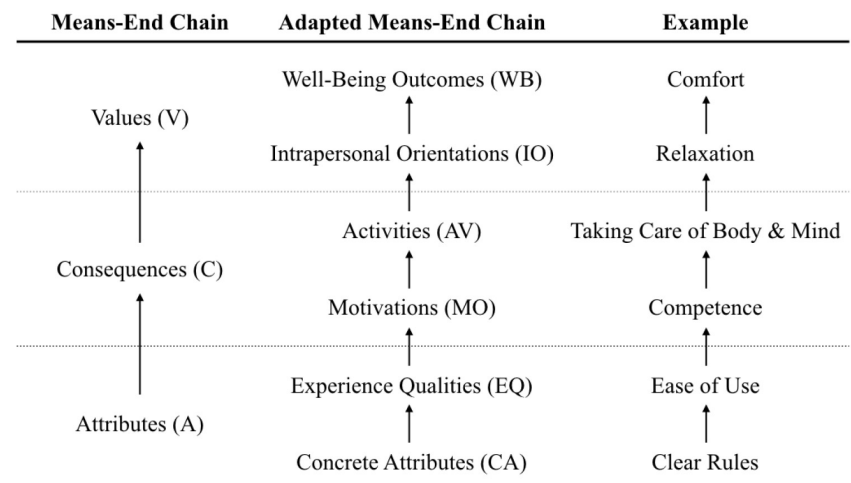

Figure 1. Means-End Chain, MEC (left), adapated MEC in the current study (middle) and example MEC (right)

Implication Matrix

Individual MECs were then aggregated across participants into an Implication Matrix (IM). This matrix reflects the number of direct and indirect links between two corresponding elements within the combined MECs. Due to the large number of categories, this step was performed using a selfprogrammed analysis software based on [50]. Separate Implication Matrices were derived for (a) all products, (b) meditation app only, and (c) personal items only. Within the scope of this paper, we focus on the overall product sample only. Aggregating data into the IM marks the transition from a qualitative (i.e., category-based) to a quantitative, pattern generating analysis technique.

Hierarchical Value Map (HVM)

In a final step, the most dominant linkages were visualized in the form of a Hierarchical Value Map (HVM), which provides a graphical representation of prominent links between attribute, consequence and value categories. To create this map, only those relations that occurred at least a minimum number of times (cutoff-level) are included. For the current 
study, a cutoff-level of three links delivered the most conclusive results with an appropriate level of granularity (i.e. neither too broad nor too narrow).

\section{RESULTS}

The primary objective of this study was to empirically explore theory-based determinants for sustained well-being from human-product-relationships (goal 1) and to investigate the role of product-mediated activities (goal 2) in this context.

\section{Goal 1: Determinants of Sustained Well-Being}

Participants were each probed about three personal products; the product sample included 36 different products: 7 wellness products (e.g. shower gel), 7 digital services (e.g. social networks), 5 gifts (e.g. necklace), 5 household items (e.g. coffee machine), 4 sport items (e.g. running shoes), 2 tech gadgets (e.g. headphones), 2 lifestyle items (e.g. Waterman pen), 2 portable devices (e.g. smartphone) and 2 other items (organizer, book).

Outcomes (i.e., values) related to short-term and sustained aspects of well-being, determinants (i.e., consequences) enabling these outcomes, and product qualities (i.e., attributes) shaping these determinants were identified based on meansend analysis, that is: statements derived from product experiences were classified as attributes (concrete attributes and experienced qualities), consequences (activities and motivations), and outcomes (intrapersonal orientations and well-being outcomes) via content analysis and linked to determinants-outcomes pathways via means-end chains and visualized in a HVM (Figure 2). As described above, connections between individual items within this map are depicted via links, with the line thickness representing the strength of the connection between two variables. Outcome items are further distinguished with regard to short-term (white) and longterm (gray) impact. Well-being outcomes, supporting activities and motivations, as well as product experience qualities that foster them are described in the following paragraphs. We further refer to ways in which concrete product attributes shape product experience qualities. Variables are defined in Figure 2 and are marked in italics in the following. Elements below the cutoff-level, i.e. mentioned less than three times, are shown with dotted outlines in the HVM. To indicate pathways including these elements, corresponding links are shown as dotted lines. All other links below the cutoff-level are not shown in Figure 2.

\section{Values: Hedonic and Eudaimonic}

Means-end analysis revealed that both hedonic and eudaimonic aspects of well-being manifest in self-reported product experiences: Of 115 product experiences, 77 (67\%) were related to hedonic and $38(33 \%)$ to eudaimonic outcomes. Hedonic outcomes were associated with feelings of comfort (56 statements; 49\%), positive emotions (17 statements; $15 \%$ ), and experiences of engagement (4 statements; 3\%). Eudaimonic outcomes were associated with aspects of psychological functioning, i.e. a sense of meaning (12 state- ments; 10\%), personal growth (11 statements; 10\%), mastery (9 statements; 8\%), strong personal relationships (4 statements; 3\%), and virtuous behavior (2 statements; $2 \%$ ). Eudaimonic outcomes were linked to intrapersonal orientations towards self-actualization (23 statements; 20\%), selfdirection ( 9 statements, $8 \%$ ), affiliation (4 statements, 3\%), and benevolence/universalism (2 statements, 2\%). Hedonic outcomes were associated with orientations towards relaxation (51 statements, 44\%), stimulation (8 statements, 7\%), hedonism (6 statements, 5\%), security/health (5 statements, $4 \%$ ), and power (4 statements, 3\%).

\section{Consequences: Activities and Motivations}

Consequences mediate the relationship between well-being outcomes and experience qualities that are malleable through design, and can be subdivided in activities and motivations. Activities and motivations identified via content analysis are summarized below and their hierarchical relationship to lowlevel product experience qualities and high-level outcomes derived from means-end analysis is shown in Figure 2.

\section{Activities}

The Activities level comprises groups of activities empirically found to be supported by products and services. The following section describes these activities and highlights links to (a) determining factors, i.e. motivations and to (b) outcomes, i.e. intrapersonal orientations and well-being aspects.

\section{Taking Care of Body and Mind}

Establishing healthy habits, routines and rituals such as exercising regularly, keeping a nutricious diet, cultivating selfcare (e.g. beauty rituals), and meditating was related to sustained well-being outcomes such as comfort and intrapersonal orientations towards relaxation and security/health (46 statements). Four pathways were particularly relevant with regard to habit-supportive design: (1) offering an opportunity for individuals to flexibly (autonomy) integrate the activity into their daily lives (context-sensitivity), (2) allowing products to facilitate continuous engagement in the activity (ease of use) and offer optimal challenges in line with people's current level of expertise (competence), (3) designing products that assign rewards in form of pleasurable (joy of use) and safe (protection) interactions as well as external feedback, and (4) fostering focused attention (concentration) on the activity.

Example: "I decided to use the [meditation] app because it is quite practical as it allows me to [meditate] when I really need it (...) and I can use it here and there (...) when I am feeling acutely overwhelmed." (participant \#3)

\section{Committing to One's Goals}

About one out of seven activities (17 statements) linked to sustained well-being were concerned with goal setting (e.g. identifying areas of personal development) and goal execution (e.g. taking concrete next steps to advance one's professional career). Well-documented in the literature [63], pursuing personal goals provides individuals with a general sense 
of purpose and meaning, helps them orient their daily lives towards these goals and ultimately fosters personal development. Goal-oriented activities were linked to well-being outcomes such as self-actualization (i.e., related to personal goals like expressing creativity, building self-confidence) and self-direction (i.e., self-directed exploration of new opportunities like taking steps to advance a professional career), which resulted in elevated feelings of meaning and mastery. In order to support the pursuit of personal goals, means-end analysis showed that products need to (1) provide feedback on goal progress (rewards $\rightarrow$ competence), (2) reduce distractions (concentration) and (3) provide activities in line with individuals' goals (autonomy) by offering relevant content (personal relevance).

Example: "Every app [on my phone] is relevant to me. I have deleted all the pre-installed apps. [They are just] a distraction (...) and our time is too precious to spend it on useless things. I have been trying for months to focus only on things that are good for me and that help me making progress in my life." (participant \#1)

\section{Managing Stress, Hardship, Trauma}

Another group of activities comprised establishing effective coping strategies (e.g. practice mindfulness, seek social support) to be able to deal with emotionally stressful situations such as mental health problems or adapting to new life circumstances (11 statements). Implementing and applying these strategies regularly resulted in more enduring feelings of comfort and inner peace. Products supported coping activities by (1) encouraging (rewards) individuals through eliciting positive emotions (joy of use, e.g. playful Headspace characters), by providing feedback on previous accomplishments (e.g. reflecting the total amount of time spent meditating) and by directly reducing tension and negative emotions (protection, e.g. headphones preventing sensory overstimulation). These rewards functioned as positive reinforcement and fostered feelings of competence. Individuals' perceived level of competence (2) was also promoted by making product interactions easy and efficient (ease of use, e.g. through clear instructions) and by suggesting optimal challenges, e.g. short duration of meditation sessions.

Example: "When I am feeling acutely tense, I also use the [short] SOS meditation sessions. It helps me to distance myself from the immediate, stressful situation." (participant \#5)

\section{Learning}

An enduring sense of personal growth was promoted by activities related to learning (e.g. tracking one's activity level and energy consumption), skill building (e.g. specific meditation techniques) and adopting new perspectives (e.g. when ruminating over a relationship problem). The most significant way products supported learning activities was by (1) making individuals feel competent through easy-to-use interfaces (ease of use) and by enabling them to adapt the difficulty of the activity (optimal challenge). In addition, products supported individuals in (2) focussing on the learning activity (concentration) by providing relevant content (personal relevance).

Example: "I started meditating for only five minutes using the 'Basics' module because meditating can be daunting at the beginning. At some point, I increased the duration to 10 minutes depending on how competent I felt. When I took a meditation class [in the past], they started with 25 minutes sessions right away which was overwhelming for me." (participant \#2)

\section{Living in the Present}

Products also supported individuals to live in the present by savoring positive and meaningful experiences (3 statements) through intensifying (e.g. adding sensory pleasures such as candles or chocolate), prolonging (e.g. functioning as a tangible symbol of a personally meaningful goal) or anticipating (e.g. pleasant voice of the meditation teacher facilitating relaxation) these experiences (see also [46]) which functioned as rewards. Savoring was linked to both hedonic and eudaimonic well-being outcomes, i.e. positive emotions, feelings of comfort and a sense of personal growth.

Example: "I put a lot of effort into making myself comfortable in my new apartment. Now I am able to relax on my couch and I regularly perform a little ceremony. I light lots of candles which gives me the feeling that I finally have a home." (participant \#7)

\section{Contributing to the Greater Good}

Virtuous behavior (virtue) concerned with the welfare of others such as sustainable and ethical consumption was linked to durable well-being ( 2 statements). This group of activities was associated with products that resonate with a person's moral values, e.g. are manufactured following decent labor standards. Acting in accordance with one's personal values promotes feelings of autonomy and increases intrinsic motivation to engage in the activity.

Example: "It's important to me that [the thermos] is made from BPA free plastic (...) and that I can always bring the thermos with me (...) and reuse it. That way I am not contributing to a throwaway society." (participant \#10)

\section{Investing in Social Connection}

Lastly, products supported individuals in strengthening social relationships with others (1 statement) by providing opportunities for social affirmation (rewards).

Example: "I upload my own photos to a photography interest group on Facebook (...). This way I am sharing my happiness with others (...) and it makes me happy to see that others enjoyed my photos." (participant \#4)

As the research focus is primarily on well-being-enhancing activities, only those motivations included in prominent MECs (cutoff value: $\mathrm{N}=3$ ) are described in more detail below, i.e., competence, rewards, autonomy, and concentration; all empirically derived motivations are listed in Figure 2 (with a short description). 


\section{Motivations}

Motivations describe psychological effects of product usage including support of well-being-enhancing activities. Product experience qualities shaping these motivations are described below and depicted in Figure 2. Links to supported types of activities are visualized in Figure 2.

\section{Competence}

The most common way for a product to support well-being activities (30 statements) was to make individuals feel capable and effective when performing an activity. Products supporting competence motivations (1) offer optimal challenges, (2) are easy to use, and (3) encourage participants to keep performing an activity through rewards (e.g. feedback on goal progress).

\section{Rewards}

Products also fostered well-being-related activities via external rewards (28 statements) and increased the likelihood that desired behaviors occur again in the future. Experience qualities linked to reward motivation include (1) joy of use (i.e., positive emotions generated through direct interaction with the product), (2) protection (i.e., reduced negative emotions), and (3) feedback (e.g. "likes" on social networks).

\section{Autonomy}

Another way products supported activities was by enabling people to act independently (26 statements) and in accordance with deeply-held personal goals and values. Experience qualities that support autonomy motivations include (1) context-sensitivity (i.e., allowing adaptation of the activity to fit into a person's daily life) and (2) personal relevance (i.e., choosing activities matching one's personal goals and values, offering relevant content and functionalities).

\section{Concentration}

In addition, products supported individuals to focus on the activity (11 statements) by reducing distractions and maintaining states of flow through providing personally-relevant content (i.e., in accordance with personal values and goals).

\section{Experience Qualities}

Experience qualities that were mentioned at least three times (cutoff-level) are described in more detail below. We further illustrate various ways in which concrete product attributes may support these experience qualities. That relationship, however, has been subject to many studies on product experience and is beyond the scope of this study. All mentioned experienced and concrete product attributes are shown in Figure 2.

\section{Personal Relevance}

One major contribution of products (19 statements) to wellbeing was to enable individuals to choose activities that match their personal goals and interests. Concrete product attributes that were found to support personally-relevant activities include (1) a variety of functions (e.g. diverse meditation packages, multiple settings), (2) relevant content and (3) opportunities for customization (e.g. deciding which apps to install on one's phone).

\section{Ease of Use}

Products further facilitated activities by making the product interaction easier and more efficient (16 statements). Ways to do so were by (1) providing structure (e.g. modular meditation packages), (2) using clear content (e.g. metaphors, instructions), and (3) defining clear rules (e.g. step-by-step introduction, takeaway messages).

\section{Joy of Use}

Products also fostered sustained well-being through joy of use including sensual delight, intellectual stimulation, and aesthetic pleasure (15 statements). Concrete product attributes increasing joy of use include (1) sensory qualities (e.g., smell, texture), (2) instilling a sense of social connectedness (e.g. friendly cartoon characters in Headspace), (3) typicality (e.g. familiar voice of the meditation teacher), (4) novelty (e.g. surprising interactions, new editions), and (5) offering a broad variety of contents and functions.

\section{Context-Sensitivity}

In order for individuals to integrate activities into their daily lives and engage in them regularly, products need to be adaptive to people's lifestyle (15 statements) by (1) offering flexible time-settings (dosage) and (2) allowing them to perform the activity when needed, such as portable items or digital services available as app (ubiquity).

\section{Optimal Challenge}

Products further supported well-being by enabling people to choose activities that match their current level of expertise thereby supporting states of flow and continued engagement in the activity ( 8 statements). One way for products to do so is by (1) offering helpful communication and an appropriate level of guidance (content; e.g. metaphors) and by (2) allowing individuals to adjust frequency and duration of the activity (dosage) according to their skill level.

\section{Protection}

Products further encouraged individuals to engage in wellbeing-increasing activities by reducing negative emotions and dispelling concerns (5 statements), e.g. ecological materials, noise-canceling headphones.

\section{Goal 2: Activities as Pathway to Sustained Well-Being}

The second goal of this research was to visualize pathways that link product experience qualities to well-being determinants and well-being outcomes, and to understand which role activities play along these pathways. 24 product experiences $(21 \%)$ were focused on direct product interactions and 19 of these experiences were limited to short-term increases of hedonic well-being (17\%), i.e. positive emotions, engagement (see Figure 2). An astonishingly large number of 96 self-reported product experiences $(83 \%)$, however, were associated with lasting increases in well-being, and 91 of these experiences $(79 \%)$ were linked to activities that extended beyond the mere product interaction. Most importantly, these activities were offered (e.g. various themes), initiated (e.g. through reminders) and/or maintained (e.g. through rewards, optimal challenges) by elements of design. The favorable long-term 


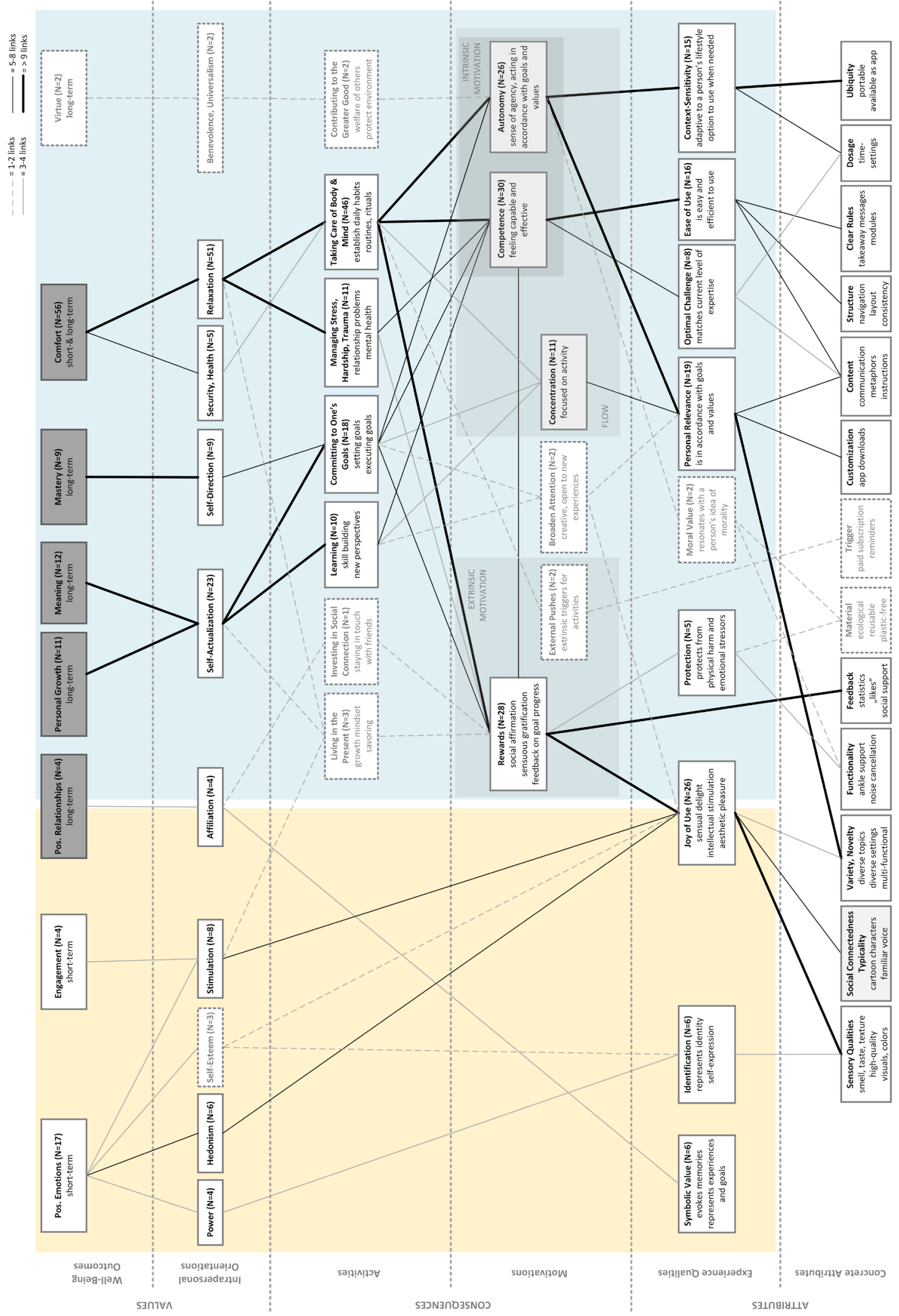

Figure 2. Hierarchical Value Map (HVM) depicting Attributes (A), Consequences (C), and Values (V). The graphic visualizes products' direct (left, yellow) and indirect contributions (right, blue) to well-being mediated via activities. Products supported short-term (white) and long-term (gray) well-being outcomes. 
impact of these activities was related to performing them regularly (e.g. habits, hobbies), their association with longerterm, meaningful pursuits (e.g. working towards personal goals, learning a new skill, shaping one's personality) and/or resulting persistent changes of one's character (e.g. self-development). A considerable number $(30 \%, \mathrm{~N}=34)$ of activity-based, lasting increases in well-being mediated by products were eudaimonic in nature, i.e. related to well-being outcomes such as personal growth, meaning, and mastery. Note that, in our sample, the majority of activities $(47 \%, N=54)$ contributing to enduring well-being were associated with daily habits (esp. regular meditation practice) leading to an increased sense of inner peace and serenity (comfort). A small number of long-term well-being outcomes were not linked to activities $(3 \%, \mathrm{~N}=4)$ but rather to symbolic representations of connectedness and personal growth conveyed by souvenirs and gifts (see also [7]).

\section{DISCUSSION}

The goal of this research was to take first steps towards a library of empirically validated determinants of sustained well-being in human-product-relationships (goal 1). We also explored the specific contribution of product-mediated activities by inspecting (causal) pathways from experience qualities to well-being outcomes derived from means-end analysis (goal 2).

\section{Goal 1: Determinants of Sustained Well-Being}

Supporting theoretical claims in well-being design [[6],[13],[27],[42],[51]], the current study provides empirical evidence that products are capable of shaping a diverse range of sustained well-being outcomes beyond temporary pleasures. Long-term outcomes comprised both hedonic and eudaimonic aspects, e.g. positive emotions, personal growth and meaning. Derived from a bottom-up-top-down-approach, determinants delineated in this paper stem from a theoretical and empirical basis. The current work thereby refines purely theory-driven recommendations (e.g. [[6],[13]]) how to design for sustained well-being. It further adds two layers of determinants not explicitly considered in previous theoretical work, i.e. experience qualities and activities. In our view, these determinants provide a particularly promising starting-point for well-being design as they are comparatively tangible and thus effectively malleable through design. In contrast, well-being design frameworks focus primarily on rather abstract motivational determinants, e.g. psychological needs [[27],[42]], personal goals [13] or rewards [6]. This being said, following (causal) pathways from experience qualities to motivations, the current study also shows how abstract determinants may be linked to specific realizations of product attributes.

The reported findings are in line with previous research in psychology and well-being design: The detected list of $\mathrm{Ac}$ tivities coincides with taxonomies of Positive Psychology Interventions (PPIs) [[17],[32]] which have empirically been linked to lasting increases in well-being (e.g., [62]). The broadest overlaps were identified with Lyubomirsky's classification of PPIs [32], i.e. 'taking care of body and mind', 'committing to one's goals', 'managing stress, trauma, hardship', 'living in the present', and 'investing in social connection' (see Figure 2). We discovered two additional types of product-mediated activities, i.e. 'learning' and 'contributing to the greater good' linked to long-term well-being outcomes. Virtuous activity, i.e. contributing to the greater good has also been linked to lasting increases in well-being in positive psychology before [[43],[60]]. Previous research found similar types of activities to be associated with hedonic and eudaimonic well-being outcomes [[27],[35]] in human-product-relationships. However, the current study illustrates specific ways how to shape these distinct activity types through design.

Motivations that were empirically derived in the current work overlap with four prevalent motivation theories: (a) intrinsic motivation [54], (b) extrinsic motivation [11], (c) flow [10], and (d) Broaden-and-Build-Theory [19]. These theoretical overlaps are indicated in Figure 2. Consistent with SelfDetermination Theory [[52],[55]] and theoretical claims in well-being design [[27],[42]], products fostered intrinsic motivation by supporting well-being-enhancing activities in a way that satisfies basic psychological needs for autonomy, competence, and relatedness (herein referred to as affiliation) [61]. Relatedness was further linked to symbolic product interactions, e.g. gifts reflecting meaningful personal relationships (see also [7]). In line with Organismic Integration Theory [11], specific types of extrinsic rewards such as feedback on goal progress and social support further promoted intrinsic motivation by increasing feelings of competence. Our findings suggest that extrinsic rewards allocated by products are particularly effective when individuals lack initial skills or deliberation (committing to one's goals), are confronted with challenging life situations (managing stress, hardship, trauma), or face difficulties integrating an activity into their daily routines and habits (taking care of body and mind). Besides intrinsic motivation, products shaped further characteristics of flow experiences [10] such as deep concentration on the task, clear rules and immediate feedback. Lastly, in line with Frederickson's [19] Broaden-and-Build-Theory, pleasure-evoking design stimulated motivation in beneficial ways by expanding users' thought-action-repertoires.

Besides well-known constituents of hedonic and pragmatic qualities [23], i.e. joy of use, identification, symbolic value, and ease of use, the list of product experience qualities includes additional variables proven to increase the efficiency of PPIs [33] and characteristics of flow experiences [10]. This indicates that existing user experience frameworks (e.g. [23]) and measurement tools (e.g. [25]) might need to be extended to incorporate all relevant categories of experienced product qualities promoting sustained well-being. Consistent with previous empirical research [35] and theoretical claims [47], our data support the involvement of pleasure-evoking design in mediating long-term hedonic and eudaimonic wellbeing outcomes. Previous studies did not find substantial 
correlations between pragmatic qualities and hedonic and eudaimonic well-being outcomes [[26],[35],[37]]. In contrast, well-being-increasing activities were substantially facilitated by pragmatic product qualities in our study. The laddering approach taken in the current study might have been particularly well-suited to capture this facilitation effect as it differentiates multiple levels of product impact and explored self-reported product experiences in more detail.

\section{Goal 2: Activities as Pathway to Sustained Well-Being}

As outlined above, participants' self-reported product experiences were linked to short-term (19 statements, 17\%) and long-term (96 statements, 83\%) well-being outcomes. Most intriguingly, sustained well-being was to a large extent mediated via activities (91 statements, 79\%). Considering the strong empirical evidence linking activities and sustained well-being in previous research (e.g. [62]) and findings from the current study, we propose to regard activities as the most essential determinant in design for sustained well-being and to focus design efforts on activities accordingly. In our view, the taxonomy of activities discussed in this paper provides a good starting point. This collection may function as an inspiration to design for the 'right' activities, i.e. those most robustly linked to lasting increases in well-being. In order to prevent detrimental effects on individual or societal well-being, this list could further be complemented with activities empirically shown to detract from well-being, e.g. unfavorable social comparisons [32].

\section{Implications for Positive Design}

The current study provides straightforward guidance for practioners to design for lasting increases in well-being. Besides streamlining the list of theoretically derived determinants, we visualized (causal) pathways from experienced product qualities to well-being determinants and well-being outcomes in a HVM. Design for long-term impact may tackle any component along these pathways. Low-level elements linked to a dedicated component constitute opportunities to shape this component through design. The strength of association between connected elements further indicates the most promising starting points for design efforts. As outlined above, we particularly like to encourage practioners to start the design process with thinking about product-mediated activities first. Ingredients on the Motivations and the Experience Qualities level can then be derived from the HVM. For example, if a product aims to support individuals in establishing effective coping strategies (i.e., managing stress, hardship, trauma), designers may preferably focus on addressing users' perceived level of competence by keeping the product interaction as simple as possible (ease of use) and by providing optimal challenges matching the person's current skills. Extrinsic rewards such as social affirmation may further increase feelings of competence. Other types of rewards, e.g. sensuous gratification represent promising levers as well but due to their weaker association with coping activities (indicated by line strength of the direct link between rewards and managing stress, hardship, trauma in Figure 2) may be targeted with a lower priority. Another example deductible from the HVM is that products should not interfere with individuals' intrinsic drive to perform well-being-enhancing activities but rather promote feelings of autonomy and competence. Effective ways to address these needs by means of design are focusing on pragmatic product qualities, (esp. ease of use, context-sensitivity) and to synchronize the product with users' personal goals and values (personal relevance).

\section{LIMITATIONS AND FUTURE WORK}

A major limiting factor of the current work is the small product and participant sample the study is based on. The deduced HVM might further overemphasize pathways related to meditation apps. Selection bias is another potential concern as participants were selected based on their previous usage of a specific meditation app. This selection might be associated with specific socio-demographic characteristics and motivational orientations within the tested sample, e.g. towards relaxation and self-actualization. Therefore, the current findings must be interpreted with caution and future research is needed to extrapolate results to a broader range of product categories and participants. It is important to note that laddering represents an established method in marketing to study consumer decision making. In HCI, laddering has been applied less frequently and with different purposes, i.e. to understand user motivation and to derive design recommendations [66] which limits the current state of knowledge regarding its validity in this context. Lastly, laddering and meansend analysis pose diverse methodological challenges including subjectivity associated with content analysis and data reduction [[21],[36],[64]]. As with any exploratory research, insights derived from this study thus need to be validated in future research.

\section{CONCLUSION}

Research in Positive Psychology has confirmed intentional engagement in specific activities to be a crucial determinant of sustained well-being. So far, design research has theoretically acknowledged the opportunity to shape well-being-enhancing activities through products and services but has not yet provided actionable guidance how to do so. The current research establishes product-mediated activities as the most crucial determinant when designing for long-term impact. It further proposes a taxonomy of activity types malleable through design and illustrates opportunities to shape these activities through specific realizations of product attributes and a range of determining factors. This research supports decision-making for practioners in industry contexts and will thus facilitate the transfer of academic contributions in wellbeing design into real-world applications.

\section{ACKNOWLEDGMENTS}

We would like to thank all participants of the study. Special thanks to eBay and brands4friends for funding this research.

\section{REFERENCES}

[1] Ida H. Bennike, Anders Wieghorst, and Ulrich Kirk. 2017. Online-based mindfulness training reduces behavioral markers of mind wandering. Journal of 
Cognitive Enhancement 1, 2: 172-181.

[2] Wendy R. Boswell, John W. Boudreau, and Jan Tichy. 2005. The relationship between employee job change and job satisfaction: The honeymoon-hangover effect. Journal of Applied Psychology 90, 5: 882-892.

[3] Philip Brickman and Donald T. Campbell. 1971. Hedonic relativism and planning the good society. In Adaptation-Level Theory: A Symposium, H. M. Apley (ed.). Academic Press, New York, NY, US, 287-302.

[4] Philip Brickman, Dan Coates, and Ronnie JanoffBulman. 1978. Lottery winners and accident victims: Is happiness relative? Journal of Personality and Social Psychology 36, 8: 917-927.

[5] Fred B. Bryant and Joseph Veroff. 2007. Savoring: A New Model of Positive Experience. Lawrence Erlbaum Associates Publishers, Mahwah, NJ.

[6] Rafael A. Calvo and Dorian Peters. 2014. Positive Computing: Technology for Well-Being and Human Potential. MIT Press, Cambridge, MA.

[7] Mafalda Casais, Ruth Mugge, and Pieter Desmet. 2016. Using symbolic meaning as a means to design for happiness: The development of a card set for designers. In Proceedings of the 2016 Design Research Society 50th Anniversary Conference (DRS '16), 1553-1571). https://doi.org/10.21606/drs.2016.424

[8] Fulvio Castellacci and Vegard Tveito. 2018. Internet use and well-being: A survey and a theoretical framework. Research Policy 47, 1: 308-325.

[9] Juliet Corbin and Anselm Strauss. 1990. Grounded theory research: Procedures, canons, and evaluative criteria. Qualitative Sociology 13, 1: 3-21.

[10] Mihalyi Csikszentmihalyi. 1990. Flow: The Psychology of Optimal Experience. Harper \& Row, New York, NY.

[11] Edward L. Deci and Richard M. Ryan. 1985. Toward an organismic integration theory. In Intrinsic Motivation and Self-Determination in Human Behavior. Perspectives in Social Psychology. Springer US, Boston, MA, US, 113-148.

[12] Pieter Desmet and Marc Hassenzahl. 2012. Towards happiness: Possibility-driven design. In HumanComputer Interaction: The Agency Perspective. Studies in Computational Intelligence, M. Zacarias and J.V. de Oliveira (eds.). Springer, Berlin, Heidelberg, 3-27.

[13] Pieter M. A. Desmet and Anna E. Pohlmeyer. 2013. Positive design: An introduction to design for subjective well-being. International Journal of Design 7, 3: 5-19.

[14] Sarah Diefenbach, Nina Kolb, and Marc Hassenzahl. 2014. The 'hedonic' in human-computer interaction -
History, contributions, and future research directions. In Proceedings of the 2014 Conference on Designing Interactive Systems (DIS '14), 305-314. https://doi.org/10.1145/2598510.2598549

[15] Marcos Economides, Janis Martman, Megan J. Bell, and Brad Sanderson. 2018. Improvements in stress, affect, and irritability following brief use of a mindfulness-based smartphone app: A randomized controlled trial. Mindfulness 9, 5: 1584-1593.

[16] Steven Fokkinga, Paul Hekkert, Pieter Desmet, and Elif Özcan. 2014. From product to effect: Towards a human-centered model of product impact. In Proceedings of the 6th Design Research Society Conference (DRS '14), 71-83.

[17] Michael W. Fordyce. 1977. Development of a program to increase personal happiness. Journal of Counseling Psychology 24, 6: 511-521.

[18] Shane W. Frederick and George Loewenstein. 1999. Hedonic adaptation. In Well-being: The Foundations of Hedonic Psychology, Daniel Kahneman, Ed Diener and Norbert Schwarz (eds.). Russell Sage Foundation, New York, NY, US, 302-329.

[19] Barbara L. Fredrickson. 2001. The role of positive emotions in positive psychology: The broaden-andbuild theory of positive emotions. American Psychologist 56, 3: 218-226.

[20] Robbie Gonzalez. 2018. Why Apple Can’t Tackle Digital Wellness In A Vacuum. Wired Magazine. Retrieved from www.wired.com

[21] Klaus G. Grunert and Suzanne C. Grunert. 1995. Measuring subjective meaning structures by the laddering method: Theoretical considerations and methodological problems. International Journal of Research in Marketing 12, 209-225.

[22] Jonathan Gutman. 1982. A means-end chain model based on consumer categorization processes. Journal of Marketing 46, 2: 60-72.

[23] Marc; Hassenzahl. 2003. The thing and I: Understanding the relationship between user and product. In Funology, Mark M. Blythe, Kees Overbeeke, Andrew F. Monk, and Peter C. Wright (eds.). Kluwer Academic Publishers, Norwell, MA, US, 31-42.

[24] Marc Hassenzahl. 2010. Experience design: Technology for all the right reasons. Synthesis Lectures on Human-Centered Informatics 3, 1: 1-95.

[25] Marc Hassenzahl, Michael Burmester, and Franz Koller. 2003. AttrakDiff : Ein Fragebogen zur Messung wahrgenommener hedonischer und pragmatischer Qualität. Mensch \& Computer 2003: Interaktion in Bewegung: 187-196.

[26] Marc Hassenzahl, Sarah Diefenbach, and Anja Göritz. 2010. Needs, affect, and interactive products - Facets 
of user experience. Interacting with Computers 22, 5: 353-362.

[27] Marc Hassenzahl, Kai Eckoldt, Sarah Diefenbach, Matthias Laschke, Eva Lenz, and Joonhwan Kim. 2013. Designing moments of meaning and pleasure. Experience design and happiness. International Journal of Design 7, 3: 21-32.

[28] Eric B. Hekler, Predrag Klasnja, Jon E. Froehlich, and Matthew P. Buman. 2013. Mind the theoretical gap: Interpreting, using, and developing behavioral theory in HCI Research. In Proceedings of the SIGCHI Conference on Human Factors in Computing Systems (CHI '13), 3307-3316.

https://doi.org/10.1145/2470654.2466452

[29] Veronika Huta. 2016. An overview of hedonic and eudaimonic well-being concepts. In Handbook of Media Use and Well-Being: International Perspectives on Theory and Research on Positive Media Effects. L. Reinecke and M. B. Oliver (eds.). Routledge, New York, NY, US, 14-33.

[30] Veronika Huta and Alan S. Waterman. 2014. Eudaimonia and its distinction from hedonia: Developing a classification and terminology for understanding conceptual and operational definitions. Journal of Happiness Studies 15, 6: 1425-1456.

[31] Richard E. Lucas, Andrew E. Clark, Yannis Georgellis, and Ed Diener. 2003. Reexamining adaptation and the set point model of happiness: Reactions to changes in marital status. Journal of Personality and Social Psychology 84, 3: 527-539.

[32] Sonja Lyubomirsky. 2007. The How of Happiness: A Scientific Approach to Getting the Life You Want. Penguin Press, New York, NY.

[33] Sonja Lyubomirsky and Kristin Layous. 2013. How do simple positive activities increase well-being? Current Directions in Psychological Science 22, 1: $57-62$.

[34] Sonja Lyubomirsky, Kennon M. Sheldon, and David Schkade. 2005. Pursuing happiness: The architecture of sustainable change. Review of General Psychology 9, 2: 111-131.

[35] Elisa D. Mekler and Kasper Hornbæk. 2016. Momentary pleasure or lasting meaning? In Proceedings of the 2016 CHI Conference on Human Factors in Computing Systems (CHI '16), 4509-4520. https://doi.org/10.1145/2858036.2858225

[36] Tânia Modesto Veludo-de-Oliveira, Ana Akemi Ikeda, and Marcos Cortez Campomar. 2006. Discussing laddering application by the means-end chain theory. The Qualitative Report 11, 4: 626-642.

[37] Livia J. Müller, Elisa D. Mekler, and Klaus Opwis. 2015. Facets In HCI: Towards understanding eudaimonic UX -- Preliminary findings. In
Proceedings of the 33rd Annual ACM Conference Extended Abstracts on Human Factors in Computing Systems (CHI EA '15), 2283-2288. https://doi.org/10.1145/2702613.2732836

[38] Arielle Pardes. 2018. Google and the Rise of "Digital Well-Being." Wired Magazine. Retrieved from www.wired.com

[39] Timo Partala and Aleksi Kallinen. 2012. Understanding the most satisfying and unsatisfying user experiences: Emotions, psychological needs, and context. Interacting with Computers 24, 1: 25-34.

[40] Timo Partala and Sari Kujala. 2016. Exploring the role of ten universal values in using products and services. Interacting with Computers. 28, 3: 311-331.

[41] Lindsey Patterson and Robert Biswas-Diener. 2012. Consuming happiness. In The Good Life in a Technological Age, Philip Brey, Adam Briggle and Edward Spence (eds.). Routledge, New York, NY, US, 147-156.

[42] Dorian Peters, Rafael A. Calvo, and Richard M. Ryan. 2018. Designing for motivation, engagement and wellbeing in digital experience. Frontiers in Psychology 9, 797.

[43] Christopher Peterson and Martin E. P. Seligman. 2004. Character Strengths and Virtues. A Handbook and Classification. Oxford University Press, New York, NY.

[44] Anna Pohlmeyer and Pieter Desmet. 2017. From good to the greater good. In Routledge Handbook of Sustainable Product Design (1st ed.), Jonathan Chapman (ed.). Routledge - Taylor \& Francis Group, London, UK, 469-486.

[45] Anna E. Pohlmeyer. 2012. Design for happiness. Interfaces 92: 8-11.

[46] Anna E. Pohlmeyer. 2014. Enjoying joy. In Proceedings of the 8th Nordic Conference on HumanComputer Interaction Fun, Fast, Foundational (NordiCHI '14), 871-876.

[47] Anna E. Pohlmeyer. 2017. How design can (not) support human flourishing. In Positive Psychology Interventions in Practice. Carmel Proctor (ed.), Springer, Cham, 235-255.

[48] Anna E. Pohlmeyer and Pieter M. A. Desmet. 2016. Positive Design. Designing the Road of Happiness. Workshop held at International Conference of Design and Emotion 2016.

[49] Jordi Quoidbach, Elizabeth V. Berry, Michel Hansenne, and Moïra Mikolajczak. 2010. Positive emotion regulation and well-being: Comparing the impact of eight savoring and dampening strategies. Personality and Individual Differences 49, 5: 368373. 
[50] Thomas J. Reynolds and Jonathan Gutman. 1988. Laddering theory, method, analysis, and interpretation. Journal of Advertising Research 28, 1: 11-31.

[51] Giuseppe Riva, Rosa M. Baños, Cristina Botella, Brenda K. Wiederhold, and Andrea Gaggioli. 2012. Positive technology: Using interactive technologies to promote positive functioning. Cyberpsychology, Behavior, and Social Networking 15, 2: 69-77.

[52] Richard M. Ryan and Edward L. Deci. 2017. SelfDetermination Theory: Basic Psychological Needs in Motivation Development and Wellness. Guilford Press, New York, NY.

[53] Richard M. Ryan, Veronika Huta, and Edward L. Deci. 2008. Living well: A self-determination theory perspective on eudaimonia. Journal of Happiness Studies 9, 1: 139-170.

[54] Richard M. Ryan and Edward L Deci. 2000. Intrinsic and extrinsic motivations: Classic definitions and new directions. Contemporary Educational Psychology 25, 1: 54-67.

[55] Richard M. Ryan and Edward L. Deci. 2000. Selfdetermination theory and the facilitation of intrinsic motivation, social development, and well-being. American Psychologist 55, 1: 68-78.

[56] Richard M. Ryan and Edward L. Deci. 2001. On happiness and human potentials: A review of research on hedonic and eudaimonic well-being. Annual Review of Psychology 52: 141-66.

[57] Carol D. Ryff. 1989. Happiness is everything, or is it? Explorations on the meaning of psychological wellbeing. Journal of Personality and Social Psychology 57, 6: 1069-1081.

[58] Carol D. Ryff and Burton H. Singer. 2008. Know thyself and become what you are: A eudaimonic approach to psychological well-being. Journal of Happiness Studies 9, 1: 13-39.

[59] Elizabeth B.-N. Sanders and Pieter J. Stappers. 2012. Convivial Design Toolbox: Generative Research For the Front End of Design. BIS, Amsterdam.

[60] Martin E. P. Seligman. 2011. Flourish: A Visionary New Understanding of Happiness and Well-Being. Free Press, New York, NY.

[61] Kennon M. Sheldon, Andrew J. Elliot, Youngmee Kim, and Tim Kasser. 2001. What is satisfying about satisfying events? Testing 10 candidate psychological needs. Journal of Personality and Social Psychology. 80, 2: 325-339.

[62] Kennon M. Sheldon and Sonja Lyubomirsky. 2006. Achieving sustainable gains in happiness: Change your actions, not your circumstances. Journal of Happiness Studies 7, 55-86.

[63] Kennon M. Sheldon and Andrew J. Elliot. 1999. Goal striving, need satisfaction, and longitudinal wellbeing: The self-concordance model. Journal of Personality and Social Psychology 76, 482-497.

[64] Elin B. Sorensen and Søren Askegaard. 2007. Laddering: How (not) to do things with words. Qualitative Market Research 10, 1: 63-77.

[65] Alexandre N. Tuch, Rune Trusell, and Kasper Hornbæk. 2013. Analyzing users' narratives to understand experience with interactive products. In Proceedings of the SIGCHI Conference on Human Factors in Computing Systems (CHI '13), 2079-2088.

[66] Vero Vanden Abeele and Bieke Zaman. 2009. Laddering the User Experience! Workshop held at User Experience Evaluation Methods in Product Development (UXEM '09). 